\title{
Principaux processus physico-chimiques et biologiques intervenant dans l'infiltration des produits polluants et leur transfert vers les eaux souterraines
}

\author{
Main physico-chemical and biological processes in pollutants percolation \\ and their transfer towards groundwater
}

par L. Chéry et C. Mouvet

Service Eau, BRGM, Orléans

Water is a transportation vector towards groundwater for various mineral and organic compounds, whatever are their origin or nature : gas, dissolved solids, colloids or particles. As the water percolates through the soil, different chemical, physical and biological processes modify the original water quality through reactions with soil, rocks and organic matter. Mechanisms occurrence and intensity vary in the three compartments of an aquiferous system : soil, unsaturated zone, and groundwater.

A large range of natural and biological processes occurs in the soil zone, together with different orders of magnitude. In the unsaturated zone instead, biological activity occurs at a lesser extent than in the soil zone, and the physical and chemical processes are also predominant. Little reactive processes take place in the saturated zone, where solution, dilution and hydrodynamic dispersion are most effective in the attenuation of contaminamts. Each of these three layers play their own role in the attenuation, the time of transfer, the propagation level and the final retention of the different transported products.

This paper presents some major geochemical processes such as adsorption-desorption, solution-precipitation reactions, oxidation-reduction phenomena, complexation affecting contaminant transport like heavy metals, organic chemicals, bacteria.

\section{I $\square$ LES TRANSFERTS DE POLLUTION}

Lorsque des contaminants se répandent dans l'environnement, la mesure des impacts potentiels nécessite de considérer à la fois la zone concernée et l'évolution de ces composés dans le milieu. Les notions de comportement, de caractéristiques physico-chimiques et de toxicité potentielle sont autant de paramètres à prendre en compte pour déterminer les investigations à réaliser, les traitements de l'eau à envisager et, plus en amont, de prévoir les zones vulnérables à certains types de pollutions.

Les pollutions des eaux souterraines peuvent être d'origine diffuse (engrais, produits phytosanitaires, apports atmosphériques), ponctuelle (stockages, décharges, industries) ou linéaire (routes, voies ferrées, cours d'eau), et avoir un caractère permanent ou accidentel. Indépendamment des mécanismes de transfert mis en jeu, il est primordial de bien connaître notamment l'origine spatiale de cette pollution (son point d'entrée dans le système aquifère) pour savoir si elle pourra atteindre les nappes. Une pollution à la surface du sol devra traverser toute une série de barrières naturelles avant d'atteindre la nappe, alors que des contaminants apportés plus en profondeur (fuite de réseaux enterrés, mise en communication par des forages non isolés de nappes superposées) auront un potentiel de contamination bien plus important.

Dans l'hypothèse de pollutions à la surface du sol, la vulnérabilité de la nappe sera conditionnée essentiellement par la protection naturelle du réservoir (couverture imperméable), le type de nappe (nappe libre ou captive), l'épaisseur et le pouvoir filtrant de la zone non saturée. Une fois la nappe atteinte, le stock d'eau souterrain et son taux de renouvellement interviennent pour conditionner le degré et l'amortissement de la pollution.

De manière générale, la propagation d'une pollution vers et dans les nappes est liée :

- à la nature du polluant,

- aux propriétés physiques du milieu,

- aux interactions possibles entre le polluant et le milieu solide (adsorption, dissolution) ou d'autres composants présents dans l'eau (complexation), 


\begin{tabular}{|c|c|}
\hline Critère de comportement & Grandeur caractéristique du produit à considérer \\
\hline Capacité à se solubiliser & $\begin{array}{l}\text { Solubilité dans l'eau en } \mathrm{mg} / \mathrm{l}=\text { masse maximale de produit qui peut se solubiliser } \\
\text { dans un litre d'eau à température ambiante. }\end{array}$ \\
\hline Ecoulement vertical du fluide & $\begin{array}{l}\text { Densité du liquide/ eau = rapport des masses volumiques } \\
\text { Viscosité dynamique : exprimée en } \mathrm{P} \text { (poise, } \mathrm{kg} . \mathrm{s} / \mathrm{cm}^{2} \text { ) ou en } \mathrm{St}\left(\mathrm{stokes}, \mathrm{cm}^{2} / \mathrm{s} \text { ) }\right. \\
\text { indique la force exercée par le fluide en opposition à son déplacement. On peut } \\
\text { définir la mobilité du fluide pollué par rapport à celle de l'eau }\end{array}$ \\
\hline Capacité à se volatiliser & $\begin{array}{l}\text { Tension de vapeur }(\mathrm{Pa}) \\
\text { Point d'ébullition en }{ }^{\circ} \mathrm{C} \text {, température d'ébullition du produit à pression atmosphé- } \\
\text { rique. } \\
\text { Constante de Henry }\left(\mathrm{Pa} \cdot \mathrm{m}^{3} / \mathrm{mol}\right) \text { : ratio des concentrations dans la phase gazeuse } \\
\text { et la phase liquide }\end{array}$ \\
\hline Capacité à se désintégrer & $\mathrm{T}$ (années) : période radioactive ou temps de $1 / 2$ vie de l'isotope \\
\hline Capacité à s'adsorber sur un solide & $\mathrm{Kd}(\mathrm{ml} / \mathrm{g})$ : coefficient de partage solide-liquide \\
\hline Migration verticale des vapeurs & $\begin{array}{l}\text { Densité de vapeur par rapport à l'air qui informe du comportement des vapeurs du } \\
\text { produit dans la zone non saturée }\end{array}$ \\
\hline Affinité avec l'eau & $\begin{array}{l}\text { Coefficient de partage carbone organique - eau (Koc le plus souvent exprimé en } \\
\text { log) quantifie la répartition du composé entre l'eau et la matière organique du solide } \\
\text { Coefficient de partage octanol - eau (Kow exprimé en log), quantifie la répartition } \\
\text { du composé entre l'eau et un solvant organique spécifique, l'octanol. }\end{array}$ \\
\hline
\end{tabular}

Tableau 1. - Grandeurs permettant de caractériser les comportements des substances polluantes (d'après [1]).

\begin{tabular}{|c|c|c|c|}
\hline TYPE DE POLLUTION & $\begin{array}{l}\text { CATEGORIE DE } \\
\text { POLLUANTS }\end{array}$ & SUBSTANCES TYPIQUES & $\begin{array}{c}\text { CMA liées aux normes } \\
\text { AEP (OMS) }\end{array}$ \\
\hline Pollutions minérales & $\begin{array}{l}\text { Eléments majeurs } \\
\text { Oligo-éléments } \\
\text { Eléments en trace, } \\
\text { métaux lourds }\end{array}$ & $\begin{array}{c}\mathrm{Cl}, \mathrm{SO}_{4}, \mathrm{NO}_{3}, \mathrm{Na} \ldots \\
\mathrm{Fe}, \mathrm{Mn}, \mathrm{Al}, \mathrm{B}, \mathrm{F}, \mathrm{NH}_{4}, \mathrm{Se}, \mathrm{Ba} \\
\mathrm{Cd}, \mathrm{Pb}, \mathrm{Zn}, \mathrm{As} \ldots\end{array}$ & $\begin{array}{l}10-100 \mathrm{mg} / \mathrm{l} \\
0,1-1 \mathrm{mg} / \mathrm{l} \\
1-10 \mu \mathrm{g} / \mathrm{l}\end{array}$ \\
\hline Pollutions organiques & $\begin{array}{c}\text { Hydrocarbures } \\
\text { Molécules simples } \\
\text { Molécules complexes }\end{array}$ & $\begin{array}{l}\text { Fioul, kérosène, huile } \\
\text { Organo-halogénés, } \\
\text { solvants chlorés } \\
\text { Phytosanitaires }\end{array}$ & $\begin{array}{c}10 \mu \mathrm{g} / \mathrm{l} \\
0,2 \mu \mathrm{g} / 1 \\
0,5-2,0 \mu \mathrm{g} / 1\end{array}$ \\
\hline Pollutions biochimiques & $\begin{array}{l}\text { Produits et déchets } \\
\text { alimentaires }\end{array}$ & Matières fermentescibles & $\begin{array}{c}\text { Normes sur MES, DBO5, } \\
\text { DCO, oxygène dissous }\end{array}$ \\
\hline $\begin{array}{c}\text { Pollutions } \\
\text { microbiologiques }\end{array}$ & $\begin{array}{c}\text { Virus, bactéries pathogènes, } \\
\text { micro-organismes }\end{array}$ & Escherichia coli... & absence \\
\hline Pollution radioactive & Radionucléides & ${ }^{3} \mathrm{H}, \mathrm{Cs}$, activité a, b, g & quelques $\mathrm{Bq} / \mathrm{l}$ \\
\hline
\end{tabular}

Tableau 2. - Principales catégories de polluants avec les concentrations maximales admissibles correspondantes (normes OMS) pour l'alimentation en eau potable. 


\begin{tabular}{|c|c|}
\hline Processus (géo)chimiques & Processus physiques \\
\hline $\begin{array}{c}\text { réactions acide-base } \\
\text { adsorption/désorption } \\
\text { complexation } \\
\text { oxydation-réduction } \\
\text { dissolution-précipitation }\end{array}$ & $\begin{array}{c}\text { diffusion/convection } \\
\text { dispersion } \\
\text { évaporation } \\
\text { filtration } \\
\text { volatilisation }\end{array}$ \\
\hline Processus biochimiques & décroissance radioactive \\
\hline synthèse cellulaire \\
biodégradation & Processus biophysiques \\
& filtration des germes pathogènes \\
& transport des germes \\
& respiration cellulaire \\
\hline
\end{tabular}

Tableau 3. - Classification des différents processus naturels contrôlant le transfert des pol-

luants vers les eaux souterraines (d'après Langmuir, 1972 - modifié [3]).
- aux mélanges avec des eaux de natures différentes (dilution).

L'extension spatiale d'un panache de pollution dépend bien évidemment de l'écoulement naturel de l'eau, qui entraîne le produit par convection, de l'hétérogénéité du milieu et de la variabilité des vitesses, qui se traduisent par la dispersion longitudinale et transversale du panache au cours de sa progression. Ces processus hydrodynamiques, valables pour toutes les catégories de polluants, ne seront pas abordés dans ce document qui ne traite, de manière très synthétique, que des principaux processus biophysicochimiques.

\subsection{La nature du polluant}

Différentes catégories de polluants peuvent être distinguées : métaux lourds (produits industriels, catalyseurs de réactions, impuretés des fertilisants, résidus miniers...), substances organiques (hydrocarbures, produits phytosanitaires, phénols, liquides organiques non miscibles à l'eau - gasoil, benzène...), substances inorganiques solubles (fertilisants), gaz solubilisés dans les pluies ou aérosols, bactéries, virus... La prise en compte de la nature des polluants est primordiale pour appréhender l'impact d'un rejet vers la nappe, car le transfert de la pollution dépendra du comportement du polluant dans le milieu, comportement lié à la nature et aux propriétés physico-chimiques du produit, et aux interactions physico-chimiques et biochimiques "polluant-milieu".

On peut ainsi lister les principales caractéristiques physico-chimiques des substances susceptibles de régir leur comportement dans les milieux naturels (Tableau 1).
La connaissance de ces grandeurs physiques permet notamment d'apprécier l'affinité du polluant pour la matrice solide, affinité qui va conditionner la migration du produit (piégeage dans des niveaux riches en matières organiques, possibilité de relargage) et sa disponibilité pour des processus comme la biodégradation. Une grandeur comme le coefficient de partage octanol/eau, qui caractérise un certain potentiel de bio-accumulation dans les organismes vivants, constitue un indicateur de toxicité potentielle des substances organiques [2].

Par ailleurs, les concentrations maximales admissibles pour l'alimentation en eau potable (AEP, normes OMS) prennent également en compte cette différence de nature des polluants, en tolérant des concentrations qui peuvent varier d'un facteur 100 en fonction du produit considéré (tableau 2).

\subsection{Les phénomènes naturels pouvant contrôler le transfert de polluants vers les eaux souterraines}

Plusieurs processus bio-physico-chimiques sont à l'origine des variations des concentrations des éléments chimiques dans l'eau souterraine (tableau 3). Ils auront évidemment un rôle variable selon la nature du polluant considéré.

\subsection{Le milieu : le système aquifère}

De manière simplifiée, le système aquifère peut être représenté par trois compartiments en continuité hydraulique (le sol, la zone non-saturée et la nappe d'eau souterraine), qui vont chacun, avec leurs caractéristiques propres, jouer un rôle dans l'atténuation et le transfert de la pollution. Un

\begin{tabular}{|c|c|c|c|c|}
\hline Milieu & \multicolumn{2}{|c|}{ Sol + interfaces } & \multirow{2}{*}{$\frac{\text { ZNS }}{\text { Roche }+/ \text { - humide }}$} & \multirow{2}{*}{$\begin{array}{c}\text { Nappe } \\
\text { Roche noyée }\end{array}$} \\
\hline Matrice physique & Terre végétale ou sol & Berge de cours d'eau & & \\
\hline $\begin{array}{c}\text { Sens principal de } \\
\text { déplacement des } \\
\text { solutés }\end{array}$ & & $\Rightarrow$ & $\downarrow$ & $\Rightarrow$ \\
\hline Echelle spatiale & \multicolumn{2}{|c|}{0,1 à $1 \mathrm{~m}$} & 1 à quelques $\mathrm{m}$ & 100 à $100000 \mathrm{~m}$ \\
\hline $\begin{array}{c}\text { Phénomènes } \\
\text { en jeu }\end{array}$ & $\begin{array}{l}\text { Biodégradation } \\
\text { Fixation végétale } \\
\text { Exportation } \\
\text { adsorption }\end{array}$ & Effet filtre des berges & $\begin{array}{l}\text { Retard, dispersion } \\
\text { biodégradation, fixation } \\
\text { minérale et organique }\end{array}$ & $\begin{array}{c}\text { Dilution, dispersion, } \\
\text { délai, biodégradation, } \\
\text { adsorption }\end{array}$ \\
\hline
\end{tabular}

1. Schématisation du transfert de composés dans les 3 compartiments du système aquifère. 
schéma applicable à des nappes alluviales ou semi-libres présente ces 3 compartiments avec quelques-unes de leurs caractéristiques importantes à prendre en compte (fig.1).

Le potentiel de contamination des eaux souterraines dépend de l'atténuation du signal polluant qui va se produire entre la source de contamination et l'aquifère.

L'occurrence des principales réactions et processus listés dans le tableau 3 varie dans les différents compartiments (fig.2) :

- la zone du sol, où les processus naturels ont la plus grande variété et la plus forte amplitude, est celle où l'atténuation du signal d'entrée est la plus forte,

- la zone non saturée, qui voit une diminution progressive des processus biologiques et une augmentation relative de l'importance des processus physico-chimiques et hydrodynamiques, introduit surtout un retard dans l'arrivée du polluant dans la nappe,

- la zone saturée, où les processus majeurs sont la dilution et la dispersion hydrodynamique (des phénomènes physicochimiques voire biologiques ne sont toutefois pas à exclure), et où l'on assiste à une atténuation de la contamination.

L'épaisseur du sol, sa texture, sa structure et sa composition physico-chimique (e.g. hydroxydes de $\mathrm{Fe}$ et $\mathrm{Mn}$ pour la fixation des métaux, matière organique pour la fixation des polluants organiques) jouent un rôle dans la limitation des transferts d'eau et de contaminants vers les compartiments sous-jacents. Le sol constitue ainsi une protection naturelle importante pour l'atténuation du processus de pollution, voire son immobilisation plus ou moins irréversible. Certaines évolutions temporelles assez rapides de sa structure (p.ex. fentes de retrait) peuvent toutefois lui enlever une bonne partie de son potentiel d'atténuation des flux polluants. Dans les conditions où le potentiel épurateur du sol est conservé, sa position d'interface lui permet de contrôler assez largement les flux (p.ex. maintien d'une microflore active pour la dénitrification); c'est notamment le cas de l'effet filtre des berges ( $\mathrm{cf} \S .2$ ).

\section{II $\square$ LES PROCESSUS BIO-PHYSICO- CHIMIQUES AFFECTANT LE TRANSFERT DES POLLUANTS VERS LES NAPPES}

Certains processus conduisent à une disparition partielle ou complète du polluant dans le système aquifère (volatilisation, dégradation), d'autres provoquent un effet retard (dissolution, adsorption, complexation), certains enfin engendrent des réactions irréversibles (fixation irréversible, dégradation complète). Ils contribuent cependant à diminuer les risques potentiels pour la nappe.

Les paramètres essentiels à prendre en compte sont ceux relatifs aux caractéristiques physico-chimiques du produit concerné (e.g. densité, viscosité, solubilité, propriétés volatiles) et ceux relatifs aux conditions environnementales (e.g. température, teneur en eau, nature du sol, perméabilité, saturation résiduelle du sol en produit organique).

\subsection{Les polluant
2.1.I. La solubilité}

La solubilité dans l'eau (et donc indirectement la mobilité) des métaux lourds est contrôlée par les anions dissous les plus abondants dans l'eau souterraine, hydroxydes, carbonates, sulfates, chlorures et sulfures. La solubilité de nombreux minéraux est par ailleurs directement liée au $\mathrm{pH}$ (exemple de $\mathrm{Fe}, \mathrm{Mn}, \mathrm{Cu}$ ) et au potentiel d'oxydo-réduction (Eh) du milieu. A pH basique, de nombreux métaux précipitent sous forme de carbonates ou d'hydroxydes, voire de sulfures lorsque les conditions du milieu sont réductrices : $\mathrm{NiCO}_{3}$ précipité à $\mathrm{pH}$ basique, se dissout à $\mathrm{pH}$ acide, $\mathrm{PbS}$ précipite à $\mathrm{pH}$ basique. Inversement, $\mathrm{As}_{2} \mathrm{~S}_{3}$ se dissout à $\mathrm{pH}$ basique.

\subsubsection{Formation de complexes}

C'est une réaction chimique qui se produit souvent entre métaux lourds et anions dissous, et qui aboutit à la formation de nouvelles espèces dissoutes, plus ou moins stables en fonction du métal et du complexant. La formation de complexes dépend notamment de la concentration en chacun des agents complexants, de la concentration des autres métaux et autres cations dans la solution, et de la température du milieu. Les principaux agents complexants sont les chlorures, fluorures, sulfates, bicarbonates et composés organiques (exemple des acides humiques). La formation de complexes joue un rôle important dans la détermination des concentrations des ions réellement actifs dans la solution ; le calcul de la spéciation aqueuse permet de prévoir les réactions pouvant affecter le métal et donc sa mobilité. L'ion métallique libre est en général l'espèce la plus toxique, même si des formes organiques (cas du mercure et du sélénium) peuvent avoir une toxicité supérieure à celle de l'ion libre.

\subsubsection{Réactions de dissolution/précipitation.}

Ce sont les réactions qui régissent la répartition des éléments entre espèces dissoutes et solides. Selon la composition de la solution, un minéral peut se dissoudre ou précipiter. Les réactions de dissolution - précipitation dépendent de la concentration de l'élément, de la concentration des autres ions dans la solution (avec une prépondérance des hydroxydes, donc du $\mathrm{pH}$ pour les métaux lourds) et dans la 


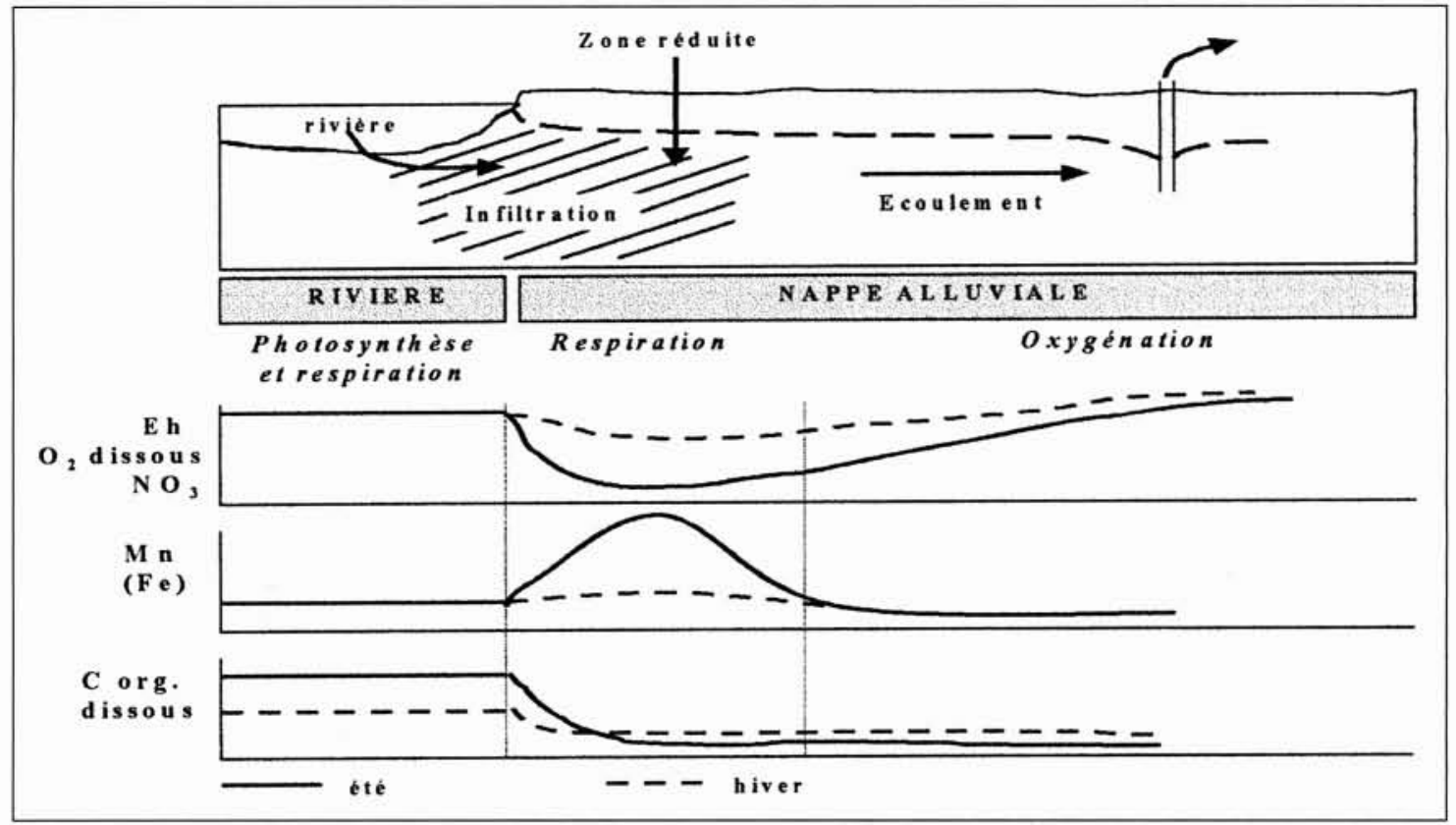

3. Effet

filtre des

berges.

Schématisa-

tion des

mécanismes

mis en jeu

(adapté de

[9]). phase solide, et de la température. Le phénomène de co-précipitation joue un rôle majeur pour les éléments traces métalliques qui peuvent ainsi être piégés dans des précipités qui impliquent à l'origine d'autres éléments : c'est le cas par exemple de $\mathrm{Cu}, \mathrm{Pb}, \mathrm{Zn}$ qui peuvent précipiter avec des hydroxydes de $\mathrm{Fe}$ ou de $\mathrm{Mn}$. La dissolution de phases solides métalliques peut être facilitée si les conditions physico-chimiques de la solution permettent la formation de complexes dissous très stables.

\subsubsection{Réactions d'adsorption-désorption}

Les ions en solution peuvent se fixer à la surface des solides par des réactions de type adsorption et/ou échange d'ions. L'adsorption se fait sur les premières couches du minéral, l'élément chimique étant fixé à la surface, contrairement au processus d'absorption où l'élément chimique est directement intégré dans la structure cristalline du minéral. Ce processus dépend de la surface des solides en contact avec l'eau (e.g. surface spécifique, point de charge nulle), et peut s'inverser lors d'un changement de conditions chimiques du milieu.

Ces réactions, qui se font préférentiellement à la surface des minéraux argileux (montmorillonite, kaolinite), de la calcite, de la silice, et des oxydes ou hydroxydes de fer et de manganèse, des colloïdes entraînent un retard, par rapport à l'écoulement de l'eau, de l'apparition, en un point d'observation donné, d'un polluant interactif.

Les réactions d'adsorption des éléments minéraux (et de certains composés organiques ionisables, comme le pentachlorophénol et certains phytosanitaires) dépendent du $\mathrm{pH}$. Même s'il est très difficile d'établir des règles générales et simples, on peut considérer que si le $\mathrm{pH}$ augmente (toutes choses égales par ailleurs), les cations s'adsorbent plus facilement alors que l'adsorption des anions diminue [6]. La fixation des anions est en général plus faible que celle des cations.

\subsubsection{Les réactions d'oxydo-réduction.}

Ces réactions jouent un rôle majeur dans l'évolution de la qualité des eaux souterraines, et sont liées souvent à l'acti- vité biologique et bactérienne du milieu. Elles correspondent à un échange d'électron entre un donneur d'électrons, appelé réducteur, et un accepteur d'électrons, l'oxydant, les deux membres de la réaction constituant un couple rédox. Des diagrammes $\mathrm{pH}-\mathrm{Eh}$, qui permettent de définir les réactions possibles et de prévoir la forme chimique sous laquelle est l'élément en solution, existent (ou peuvent être établis) pour un grand nombre de composants [7].

Si elles ne sont pas catalysées, par exemple par une activité bactérienne, ces réactions sont naturellement lentes. Par contre, si la réaction fait entrer en jeu un catalyseur (biologique ou chimique), la cinétique peut être rapide, voire presque instantanée (exemple de certains processus de dénitrification, de la réduction du fer, de la réduction des sulfates). Dans le cas d'une intervention biologique, la réaction peut être irréversible (du moins jusqu'à un autre changement du milieu) puisque les organismes n'utilisent, pour se développer, que l'un des deux membres du couple rédox. En contexte cristallin, l'origine naturelle des métaux lourds dans les nappes est souvent associée à l'oxydation de minéraux sulfurés de la roche.

L'exemple de l'effet filtre des berges montre l'impact de ces réactions d'oxydo-réduction (fig.3). La minéralisation de la matière organique au niveau de la berge entraîne la consommation de l'oxygène dissous, puis des nitrates, accompagnée d'une baisse du potentiel rédox. Il s'ensuit une mobilisation du manganèse (et/ou du fer), mobilisation favorisée par les températures élevées de la rivière en été et à l'automne. Lorsque le carbone organique bio-dégradable est consommé, la nappe est réoxygénée par l'infiltration d'eau depuis la surface du sol, ce qui s'accompagne de la précipitation du manganèse et/ou du fer qui avaient été mobilisés dans la zone réduite. Il faut donc éviter l'implantation de forages dans la zone réduite et favoriser la réoxygénation de la nappe [8]. Cet effet filtre des berges n'a toutefois pas une pérennité assurée, l'évolution du milieu, naturelle (saturation des sédiments) ou anthropique (intervention par dragage sur les berges), pouvant entraîner une remobilisation des polluants.

La chimie de l'eau joue donc un rôle capital dans le transfert vers les nappes des métaux issus d'une pollution, avec, 


\begin{tabular}{|c|c|c|c|}
\hline $\begin{array}{c}\text { Micro-organismes } \\
\text { (ou constituant) }\end{array}$ & Taille & Milieu poreux & $\begin{array}{c}\text { Diamètre équivalent } \\
\text { de pores }\end{array}$ \\
\hline Protozoaires & $10-100 \mu \mathrm{m}$ & gravier & $>400 \mu \mathrm{m}$ \\
\hline Bactéries & $0,2-5 \mu \mathrm{m}$ & sable & $12-400 \mu \mathrm{m}$ \\
\hline Virus & $20-250 \mathrm{~nm}$ & limon & $0,4-12 \mu \mathrm{m}$ \\
\hline Macromolécules & $1-10 \mathrm{~nm}$ & $\begin{array}{c}\text { Sables limoneux } \\
>33 \% \text { limons }\end{array}$ & $10 \mathrm{~nm}-10 \mu \mathrm{m}$ \\
\hline
\end{tabular}

Tableau 4. - Comparaison des tailles de bactéries, virus et molécules avec des diamètres équivalents de pores (d'après [14]).

par ordre de priorité décroissante, les paramètres suivants : $\mathrm{pH}$, conditions rédox, présence de particules solides avec des capacités d'adsorption (collö̈des), concentration en agents complexants minéraux et organiques, température...

\subsection{Les polluants organiques}

En plus des processus évoqués ci-dessus pour les éléments minéraux, certains processus spécifiques jouent un rôle important pour les molécules organiques.

\subsubsection{La biodégradation}

C'est la transformation d'une molécule (d'origine naturelle ou anthropique) en produits plus simples, pouvant aboutir à une destruction complète (minéralisation) de la molécule. La dégradation par des microorganismes va dépendre essentiellement de la densité des organismes dans les sols et les eaux, de la présence d'oxygène ou autres accepteurs d'électrons $\left(\mathrm{NO}_{3}, \mathrm{SO}_{4}\right)$, de la présence de nutriments, de la température, et des caractéristiques intrinsèques des substances polluantes (solubilité, sorption) qui, en interagissant avec le milieu, vont gouverner leur accessibilité pour les organismes vivants. Pour la plupart des polluants organiques (d'origine industrielle ou agricole), l'efficacité de la biodégradation comme mécanisme susceptible de diminuer les flux polluants va également dépendre de l'historique du milieu et des souches qui y sont présentes. Des expositions antérieures au polluant considéré entraînent souvent (par sélection/adaptation des souches) une augmentation de l'aptitude des souches à dégrader ce polluant lors d'expositions ultérieures. Cette observation a notamment été faite sur certains parcelles agricoles où l'atrazine, herbicide du maïs, avait été largement utilisée depuis de nombreuses années.

\subsubsection{La volatilisation.}

Ce mécanisme consiste en un changement de phase qui se caractérise par le fait que le compartiment gazeux est le récepteur. Le compartiment source peut être très diversifié : la surface du sol (déversement accidentel de produits industriels ou application de produits phytosanitaires), des horizons profonds du sol, de la zone non saturée, voire de l'eau souterraine (composés organiques volatiles - benzène, hydrocarbures chlorés - introduits accidentellement en profondeur), la surface des plantes (traitements foliaires par des phytosanitaires), les eaux superficielles...

Contrairement à la biodégradation, la volatilisation ne constitue qu'un changement de phase et pas une destruction du polluant ; c'est un processus qui ne fait que déplacer/décaler les problèmes. Dans le cas des phytosanitaires par exemple, il est maintenant bien établi que les eaux de pluie peuvent, pour certaines molécules, présenter des niveaux de contamination égaux, voire bien supérieurs, à ce qu'on observe dans les eaux souterraines ([10], [11], [12]).

\subsection{Les polluants microbiologiques}

Le transfert des bactéries et virus dans les sols est limité d'une part par l'adsorption, d'autre part par la filtration.

\subsubsection{L'adsorption.}

L'adsorption des bactéries et virus est très rapide (quelques heures), les virus étant plus fortement adsorbés sur des colloïdes et l'adsorption augmentant en général quand la teneur en matière organique augmente ou le $\mathrm{pH}$ diminue. Les virus ne sont pas fixés de façon irréversible, des variations de $\mathrm{pH}$ ou une forte pluie pouvant entrainer leur relargage [13].

\subsubsection{La filtration}

Le transfert en profondeur des micro-organismes étant limité si la taille des pores du milieu est comparable à celle des micro-organismes (tab.4), le processus de filtration sera bien plus efficace dans des limons que dans des sables grossiers. Par ailleurs, le mécanisme de filtration est bien évidemment lié aux conditions hydrauliques.

\section{III $\square$ CONCLUSIONS}

La contamination des aquifères par des pollutions de diverses origines est une préoccupation majeure pour les gestionnaires de la ressource en eau. Des processus bio-physico-chimiques peuvent, dans une certaine limite, constituer des barrières de sécurité qui vont atténuer ou retarder (plus ou moins longtemps selon la nature du polluant et du milieu) le transfert des flux polluants vers les eaux souterraines. Ces processus sont très diversifiés et s'expriment à des degrés divers dans les différents compartiments des systèmes aquifères. La généralisation des mécanismes mis en jeu est donc très difficile, et une très bonne connaissance d'un certain nombre de caractéristiques de la molécule et du milieu (tant son état initial que son évolution probable) est nécessaire pour une gestion optimale de la ressource.

La complexité des modes de transfert et la durée que peuvent prendre ces transferts de solutés vers et dans le milieu souterrain rendent ardues les prévisions nécessaires à la gestion à long terme de la ressource en eau. Cette gestion nécessite donc encore de nombreux travaux de recherche sur les transferts à différentes échelles, laboratoire ainsi que sites expérimentaux, avant qu'on puisse envisager une modélisation prédictionnelle. 


\section{Eau, ressources, aménagements, environnement}

\section{BIBLIOGRAPHIE}

[1] Pellet M. (1994) - Polluants organiques courants : caractéristiques physico-chimiques et comportement dans le milieu naturel. Rapport ANTEA A00495, 37p.

[2] Dabene E., MARIE F, et SMITH C. (1995) - Substances actives phytosanitaires. Caractéristiques utiles pour l'évaluation du comportement de quelques substances actives dans I'environnement. Rapport du Ministère de l'Agriculture, de la Pêche et et de l'Alimentation, Direction de l'Espace Rural et de la Forêt. 30 p. + 135 fiches synthétiques.

[3] MATthess G. et MiL.er J.C. (1994) - Chemical and biological contaminants and their subssurface behavior. In Guidebook on Mapping Groundwater Vulnerability, Vrba J. and Zaporozec Eds, IAH, Verlag Heinz Heise, vol.16.

[4] FosTER SSD (1987) - Fundamental concepts in aquifer vulnerability, pollution risk and protection strategy. In vulnerability of soil and groundwater to pollutants, TNO committee on hydrological Research. The Hague, proceedings and information, $n^{\circ} 38,69-86$.

5] GoLwER A. (1983) - Underground purification capacity, In groundwater in water resources planning. Proc. UNESCO Int. Symp. UNESCO/ IAH/IAHS, national committee of the federal republic of germany for IHP, vol.II, 1063-1072

[6] Bourg A. (1986) - Metals in aquatic and terrestrial systems : sorption. speciation and mobilization. Note technique BRGM n ${ }^{\circ} 86 / 20.56$.
[7] MiCHARD G. (1989) - Equilibres chimiques dans les eaux naturelles Publisud, 357p

[8] Garcia B., Bertin C., Ricard J., Bourg A., Lavandier P., Labroue L. (1994) - Effet de berge, effet de vase, deux facteurs différents de mobilisation du manganèse : un exemple dans un champ captant de la vallée du Lot (France). Annls Limnol., 30,1 ,67-85.

[9] Bourg A. (1992) - Effet filtre des berges et qualité de l'eau pompée en nappe alluviale. Courants, 14, 32-36.

[10] Chevreull M. et Garmouma M. (1993) - Occurrence of triazines in the atmospheric fallout on the catchment basin of the river Marne. Chemosphere, 27 (9), 1605-1608.

[11] Nations B.K. et Hallberg G.R. (1992) - Pesticides in lowa precipitations. Journal of Environmental Quality, 21, 486-492.

[12] Richards R.P., Kramer J.W., Baker D.B. et Krieger K.A. (1987) Pesticides in rainwater in the northeastern United States. Nature, 327, 129-131.

[13] MOREAU R. (1980) - Ecologie microbienne et hygiène du milieu souterrain. Actes du ler colloque national sur la protection des eaux souterraines karstiques, Besançon.

[14] Matthess G., Pekdeger A., Schróter J. (1988) - Persistence and transport of bacteria and viruses in groundwater. A conceptual evaluation. J.Contaminant Hydrology, 2, 171-188. 